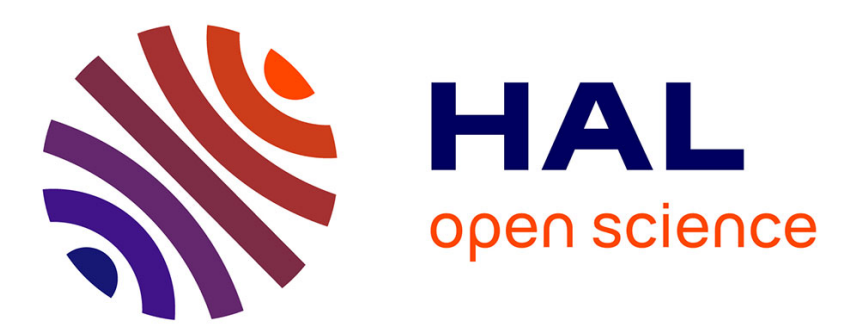

\title{
A domain decomposition method for studying the effects of missing fasteners on the behavior of structural assemblies with contact and friction
}

Laurent Champaney

\section{- To cite this version: \\ Laurent Champaney. A domain decomposition method for studying the effects of missing fasteners on the behavior of structural assemblies with contact and friction. Computer Methods in Applied Mechanics and Engineering, 2011, 10.1016/j.cma.2011.04.008 . hal-00605962}

\section{HAL Id: hal-00605962 \\ https://hal.science/hal-00605962}

Submitted on 5 Jul 2011

HAL is a multi-disciplinary open access archive for the deposit and dissemination of scientific research documents, whether they are published or not. The documents may come from teaching and research institutions in France or abroad, or from public or private research centers.
L'archive ouverte pluridisciplinaire HAL, est destinée au dépôt et à la diffusion de documents scientifiques de niveau recherche, publiés ou non, émanant des établissements d'enseignement et de recherche français ou étrangers, des laboratoires publics ou privés. 


\title{
A domain decomposition method for studying the effects of missing fasteners on the behavior of structural assemblies with contact and friction
}

\author{
L. Champaney* \\ LMT Cachan (ENS Cachan/CNRS/UPMC/PRES UniverSud Paris) \\ 61 av. du Pt Wilson - 94235 Cachan cedex - France
}

\begin{abstract}
The aim of the present work is to present an efficient numerical tool for the simulation of the behavior of 3D assemblies of structures with contact and friction when strong structural modifications occur. The paper focuses on the effects of missing connection elements (screws, bolts, rivets, ...) on the behavior of the assembly. A fast and efficient domain decomposition strategy for predicting the responses of an assembly for different configurations of missing elements is proposed. The efficiency is provided by the use of a pre-calculated solution as initialization for the calculation of a new design configuration. Applications are concerned with bolted joints under quasi-static loads.

Keywords: Missing Fasteners, Contact, Friction, Uncertainties, Assemblies, Optimization
\end{abstract}

\section{Introduction}

Many mechanical connections are made of multiple fastener assemblies so that the load is distributed on the different connecting elements. Transmission of forces in such assemblies is allowed by both friction forces due to pre-strain of the connectors and contact forces on the body of the fasteners (screws, bolts or rivets). The load capacity of the connection strongly depends on the way

\footnotetext{
* Corresponding author

Email address: laurent.champaney@lmt.ens-cachan.fr (L. Champaney )
}

Preprint submitted to Elsevier

July 5, 2011 
forces are distributed on each one of the fasteners. It is extremely important to be able to establish this distribution at the design stage. Moreover, being able to predict modifications of force distributions due to a change of state of the connection (loss of adherence, loosening of bolts, ...) or imperfections with respect to a nominal configuration (imperfect hole clearance, imperfection of friction properties, missing fasteners, ...) is also of great importance.

There is an extensive literature on the behavior of multi-fastener assemblies for nominal perfect geometries and given state of the connections. Analytical works $[1,2,3]$ offer some quick evaluation techniques for establishing the load distribution in the assemblies. In more recent works [4], finite element calculations are used to optimize the load transfer in such connections. In order to precisely establish the load transfers, many simulations use 3D finite element models. They include a fine description of frictional contact zones. Simulations using such models under quasi-static loadings are obviously not straightforward and can be time consuming. Being able to carry out such simulations is still a challenge.

The effects of friction on the load capacities and the behavior of bolted joints have been intensely studied and a review is presented in [5]. Loosening of the fasteners can have critical effects on the distribution of friction forces and so on the distribution of loads on the fasteners. The effects of such phenomena have been studied by many authors in the last decade using either experimental or numerical approaches $[6,7,8,9]$.

For assembling purposes, gaps (hole clearances) must be introduced between the assembled parts and the fasteners. Due to machining, the diameters and positions of the holes can be imperfect and so the gaps can be strongly uncertain. The effect of uncertainties of the geometry (hole clearance for example) on the behavior of the assembly has been studied in very few experimental works [10].

The simulation of the effects of all the types of imperfections and changes of state is still a challenge. The approach that is presented in this paper has been successfully used for the prediction of the effects of imperfections of pre-strain of bolts and of friction effects $[11,12]$. It has recently been extended to the 
study of imperfections of hole clearances [13].

This paper deals with the study of the effects of missing fasteners on the behavior of connections. When the number of fasteners is high, it can frequently happen that a fastener is forgotten when the connection is assembled. Moreover, loosening of a bolt can end by a complete disappearance of the bolt. Some experimental studies [14] offer some procedures for the detection of missing connectors but no specific work on numerical simulations is available. Predicting the effect of a missing fastener might be trivial as one has just to simulate the behavior of the connection without modeling the missing component. When the number of fasteners is elevated, this can lead to a large number of different simulations. This paper details a computational strategy for an accelerated determination of the solutions of such simulations.

The proposed strategy relies on a specific approach that has been developed at Laboratory of Mechanics and Technology (LMT Cachan, France) during the last twenty years and which is dedicated to the simulation of the behavior of 3D assemblies of structures. This calculation technique is based on a decomposition of the assembly that emphasize the connections by modeling them by an interface behavior. The resolution scheme is the LArge Time INcrement (LATIN) Method [15]. The approach builds, at each iteration, an approximate solution of the problem on the whole studied time interval. Its efficiency as already been proved on the calculation of solutions for quasi-static analysis of 3D assemblies of structures including a large number of frictional contact zones [16]. This approach is highly parallel and its extension to dynamics on parallel computers is presented in [17].

This approach has successfully been developed for taking into account the influence of uncertainties on connection parameters such as friction coefficients and pre-strains [11]. The main idea is the reusing of one solution that was calculated for a set of design parameters to initialize the calculation for a new set of parameters. When the responses that are obtained for both sets of parameters are close, this initialization allows a great reduction of the computational costs 
for the second calculation. When, due to the nonlinear behavior of the assembly, the two solutions are not so close, the reduction of the computational costs is still significant. To achieve the initialization, the design parameters are simply changed along the iterations in order to efficiently build solutions by re-using the best pre-calculated solutions for different sets of parameters [12].

In this paper, the proposed approach is extended to the quick calculation of a series of design configuration of assemblies where fasteners are successively missing. The purpose of the strategy that is proposed here is to get fine informations on the effects of the lack of a connecting element. In the situations that are studied here, one only seeks for the force distributions in the connecting elements before any degradation occurs in the assembly. So, only small displacements and deformations are considered with no plasticity or damage. An accurate description of the contact and friction interactions is needed in order to get the load distribution in the connectors. Obtaining this level of accuracy is only possible if conformal meshes are used on the contact zones. However, taking non-conformal meshes into account is possible ; this procedure is not presented here but is detailed in [16].

The resolution scheme and the way Coulomb's friction contact condition are taken into account are first presented. The principles of reusing solutions for initialization is detailed. Then the way the lack of fasteners is taken into account is shown. Two examples show the efficiency of the approach and some possible improvements that concern the selection of solution for initialization.

\section{Resolution scheme: LATIN method}

A brief presentation of the main aspects of the LATIN method is proposed here after. Details of the method are available in [15] and the application to the treatment of frictional contact problems are proposed in [16]. In this paper, we address structural mechanics problems including unilateral contact and friction. That is to say that we need to solve systems of nonlinear partially differential equations in time and space. Here, a discrete formulation of the LATIN method 
is presented only. It is obtained using a classical finite element discretization and time discretization of the continuous formulation that can be found in [12], which also gives the details of the discretization issues.

\subsection{Decomposition of an Assembly}

The model of structural assembly that is used here is composed of substructures $\Omega_{E}$ that communicate with each other through interfaces (see figure 1). Substructures are the components of the assembly and interfaces model the connections between the components. In some cases, substructures model sub-parts of one component. In this paper, we only consider friction unilateral contact interfaces with Coulomb's friction law. The two substructures that are connected by interface $\Gamma^{E E^{\prime}}$ are denoted $\Omega_{E}$ and $\Omega_{E^{\prime}}$.

On one interface are defined four fields. Two force fields, represented by vectors $F_{E E^{\prime}}$ and $F_{E^{\prime} E}$, model the action of the interface on each substructure. Two dual velocity fields, represented by vectors $\dot{U}_{E E^{\prime}}$ and $\dot{U}_{E^{\prime} E}$, model the motion of each substructure seen from the interface (figure 1).

\subsection{Problems on the substructures}

Classical finite element meshes are used on each substructure, and the different meshes match on the interfaces (figure 2). In the following, vectors $U$

refers to nodal displacements values, vectors $\dot{U}$ to the nodal velocity values and vectors $F$ to nodal forces.

A substructure $\Omega_{E}$ must cope with the velocity field $\dot{U}_{E \Gamma}$ of its neighboring interfaces at each time step:

$$
\dot{U}_{E \Gamma}=\mathbf{C}_{E \Gamma} \dot{U}_{E},
$$

where subscript ${ }^{E \Gamma}$ collects all the neighboring interfaces of a substructure $E$. $U_{E}$ is the displacement vector of the whole substructure. $\mathbf{C}_{E \Gamma}$ is a boolean matrix mapping the substructure degrees of freedom on the boundary.

The same substructure must also satisfy to the equilibrium equation when subject to forces due to the neighboring interfaces $F_{E \Gamma}$, and to the given body 
forces $F_{E}^{d}$, at each time step:

$$
\mathbf{K}_{E} U_{E}=F_{E}^{d}+\mathbf{C}_{E \Gamma}^{T} F_{E \Gamma}
$$

where superscript $T$ denotes transposition.

\subsection{Problems on interfaces}

At each time step, the equilibrium of the interface $\Gamma_{E E^{\prime}}$ between substructures $E$ and $E^{\prime}$ is enforced by the equation:

$$
F_{E E^{\prime}}+F_{E^{\prime} E}=0
$$

and a constitutive equation in which the forces depend on the displacement discontinuity:

$$
F_{E E^{\prime}}=f\left(U_{E^{\prime} E}-U_{E E^{\prime}}\right)
$$

where the function $f$ can be nonlinear. This function is used to describe the nonlinear behavior of the connection that is modeled by the interface.

Subscript $E E^{\prime}$ refers to the side of substructure $E$ along the interface $\Gamma_{E E^{\prime}}$; especially $F_{E E^{\prime}}$ (respectively $F_{E^{\prime} E}$ ) is the action of the same interface on substructure $E$ (resp. on the substructure $E^{\prime}$ ). The examples that are proposed in this paper only use compatible meshes on the interfaces (figure 2). The interface is composed of pairs of nodes each of them belonging to one of the opposite substructures. The nodal values in the velocities and forces vectors are ordered such that the interface equations can be written under a vectorial form.

Note that subscript $E \Gamma$ refers to a data structure that is related to each substructure independently, while subscripts $E E^{\prime}$ and $E^{\prime} E$ refer to the same quantities, but related to an interface-oriented data structure. Obviously, data transfers are required between the two descriptions. Each of these two descriptions will be used in the following, depending if the expressions are related to a substructure or an interface. 


\subsection{The iterative scheme}

The iterative scheme that is used here is derived from the LATIN method proposed by Pierre Ladevèze [15]. The main principle is to separate the equations in order to avoid the solving of both a global and a nonlinear problem. As the behaviour of the material is linear, a solution is completely given by the boundary displacement and force vectors on each substructure or by the interface displacement and forces on each interface: $s=\bigcup_{E}\left(\dot{U}_{E \Gamma}, F_{E \Gamma}\right)=$ $\bigcup_{\Gamma_{E E^{\prime}}}\left(\dot{U}_{E E^{\prime}}, F_{E E^{\prime}}, \dot{U}_{E^{\prime} E}, F_{E^{\prime} E}\right)$. The solution procedure is to search solutions $s$ that will alternatively satisfy the global linear equations (kinematic admissibility and equilibrium on the substructure) and then to the local equations (interface equations). This leads to a decoupling of the problem.

This procedure can be summarized on Figure 3 for which $s_{n}$ is the solution that satisfies to the global equations at iteration $n$ and $\widehat{s}_{n+\frac{1}{2}}$ is the one that satisfies to the interface equations between iterations $n$ and $n+1$ (subscript $n+\frac{1}{2}$ is given away in the following). The solution $s_{n}$ lies in a subspace represented by a straight line because it corresponds to linear equations. The solution $\widehat{s}$ lies in a subspace represented by a curved line because the interface behavior can be nonlinear for more complex connection conditions.

Searching for $s_{n}$ is called the linear stage, and searching for $\widehat{s}$, the local stage. At each stage, the solutions are found thanks to local and linear search directions $E^{-}$and $E^{+}$, represented by straight lines on Figure 3 .

\subsubsection{Local stage}

At this stage, a solution $\widehat{s}$ is searched from the known solution of the previous linear stage $s_{n}$ and with the upward search direction $E^{+}$. This search direction is defined interface by interface as for a augmented Lagrangian procedure:

$$
\widehat{F}_{E E^{\prime}}=F_{E E^{\prime}}+k\left(\widehat{\dot{U}}_{E E^{\prime}}-\dot{U}_{E E^{\prime}}\right)
$$

and

$$
\widehat{F}_{E^{\prime} E}=F_{E^{\prime} E}+k\left(\widehat{\dot{U}}_{E^{\prime} E}-\dot{U}_{E^{\prime} E}\right)
$$


where $k$ is a scalar parameter. As for augmented Lagrangian formulations, the rate of convergence may depend on this parameter, but the final solution does not.

The constitutive relations of the interface for a perfect connection behavior are:

$$
\widehat{\dot{U}}_{E E^{\prime}}=\widehat{\dot{U}}_{E^{\prime} E} \quad ; \quad \widehat{F}_{E E^{\prime}}+\widehat{F}_{E^{\prime} E}=0 .
$$

Using the search direction, the interface vectors can thus be computed as:

$$
\widehat{F}_{E E^{\prime}}=-\widehat{F}_{E^{\prime} E}=\frac{1}{2}\left(F_{E E^{\prime}}-F_{E^{\prime} E}\right)-\frac{1}{2} k\left(\dot{U}_{E E^{\prime}}-\dot{U}_{E^{\prime} E}\right)
$$

and

$$
\widehat{\dot{U}}_{E E^{\prime}}=\widehat{\dot{U}}_{E^{\prime} E}=\frac{1}{2}\left(\dot{U}_{E E^{\prime}}+F_{E E^{\prime}}\right)-\frac{1}{2 k}\left(F_{E E^{\prime}}+F_{E^{\prime} E}\right) .
$$

The resolution of the local stage for frictional contact behavior is presented later in the paper.

\subsection{Discretization}

Due to the form of the search directions (5) and (6), a discretization of the interface forces is also adopted. The same finite element interpolation functions are used for both the velocities and the forces on each side of one interface. Thus,

the force vectors $\widehat{F}_{E E^{\prime}}$ and $\widehat{F}_{E^{\prime} E}$ contain the nodal values of the components of the forces. On substructure $E, F_{E \Gamma}$ is a vector of finite element generalized forces that is an assembly of the forces coming from each interface. So, $F_{E \Gamma}$ is linked to the boundary force vectors $\widehat{F}_{E E^{\prime}}$ by a unit interface mass matrix.

\subsection{Linear stage}

At this stage, a solution $s_{n}$ is searched from the known solution of the previous local stage $\widehat{s}$ and with the downward search direction $E^{-}$. On each interface, this search direction is conjugate to the previous one:

$$
F_{E E^{\prime}}=\widehat{F}_{E E^{\prime}}-k\left(\dot{U}_{E E^{\prime}}-\widehat{\dot{U}}_{E E^{\prime}}\right) .
$$

Assembled on the neighboring interfaces on substructure $E$, it leads to:

$$
F_{E \Gamma}=\widehat{F}_{E \Gamma}-k\left(\dot{U}_{E \Gamma}-\widehat{\dot{U}}_{E \Gamma}\right) .
$$


The global linear system to solve at this stage is obtained with this search direction (11), the boundary conditions (1) and the equilibrium of substructure $E(2)$ :

$$
\mathbf{K}_{E} U_{E}+\mathbf{C}_{E \Gamma}^{T} k \mathbf{C}_{E \Gamma} \dot{U}_{E}=F_{E}^{d}+\mathbf{C}_{E \Gamma}^{T}\left(\widehat{F}_{E \Gamma}+k \widehat{\dot{U}}_{E \Gamma}\right) .
$$

This problem is of first order in time. It is solved at each time step starting from initial conditions by the use of an implicit integration scheme. Finally, this gives the boundary velocity $\dot{U}_{E \Gamma}=\mathbf{C}_{E \Gamma}^{T} \dot{U}_{E}$ and the boundary forces using the downward search direction at each time step.

\subsection{The frictional contact interface}

Contact problems are characterized by constraints such as non-penetration conditions, and an active area of contact - that is, an area where contact effectively occurs - that is unknown a priori. For these reasons, the problems lead to stiff non-linear systems of equations. Several approaches exist for solving static contact problems $[18,19,20]$. In most of them, the numerical methods that are employed for enforcing the contact constraints can be grouped into Lagrange multiplier and penalty methods. The penalty methods [21, 22] are closely related to the regularization of the contact constraints. They are usually formulated in terms of the displacement variables, and therefore are primal methods. They allow treating contact as a material behavior, as exemplified by the method of joint finite elements [23]. Penalty methods can experience various numerical difficulties, especially ill-conditioning, when a too large or too small penalty parameter is introduced. Lagrange multiplier methods are dual methods where the multipliers, which represent the contact reaction forces, are introduced in order to enforce exactly the non-penetration conditions. Augmented Lagrange multiplier methods $[24,25,26,27,28]$ result in mixed formulations involving both displacement and force unknowns. The numerical solution schemes underlying both the Lagrange multiplier and augmented Lagrange multiplier methods are often related to the Uzawa algorithm $[29,30]$.

Considering the strategy that was presented in the previous sections, the approach that is proposed here is a mixed domain decomposition method for a 
contact and friction problem of Augmented Lagrangian type. A dual domain decomposition method for the same type of problems was recently proposed in [31]. Moreover, some recent examples of domain decomposition strategies for contact problems are proposed in the following papers [32, 33, 34].

The following sections describe the form of function $f$ in the case of classical Coulomb's frictional contact conditions. Considering that the contact status (closed, open, sticking, sliding) can be different on each point of the interface, the local stage is solved node by node. As compatible meshes are used on the interfaces (figure 2), one point corresponds to two opposite nodes. $W_{E E^{\prime}}$ is the three-component displacement vector that is the restriction of the boundary displacements to the considered node on substructure $E$ side and $W_{E E^{\prime}}$ on substructure $E^{\prime}$ side. $T_{E E^{\prime}}$ and $T_{E^{\prime} E}$ are the three-component force vectors that is the restriction of the boundary forces to the same nodes.

In order to separate the normal behavior and the tangential one, local coordinates are used. The outward local normal at each point of an interface is defined by the three-component vector $N . N$ is oriented from substructure $E$ towards substructure $E^{\prime}$. The $3 \times 3$ projection operator on the local tangential plane $P$ is defined by :

$$
P W=W-\left(N^{T} W\right) N .
$$

For the definition of contact and sliding conditions, the jump in displacement on the interface is:

$$
D_{E E^{\prime}}=\left(W_{E^{\prime} E}-W_{E E^{\prime}}\right)
$$

\subsubsection{The unilateral contact problem}

At each time step, the non-penetrating contact conditions are:

- Open: if $N^{T} \widehat{D}_{E E^{\prime}}>0$ then $N^{T} \widehat{T}_{E E^{\prime}}=N^{T} \widehat{T}_{E^{\prime} E}=0$,

- Contact: if $N^{T} \widehat{D}_{E E^{\prime}}=0$ then $N^{T} \widehat{T}_{E E^{\prime}}=-N^{T} \widehat{T}_{E^{\prime} E} \leq 0$.

These conditions can be simply written as:

- Open: $c_{n}>0$, 
- Contact: $c_{n} \leq 0$,

where the contact indicator $c_{n}$ is:

$$
c_{n}=\frac{1}{2} N^{T} \widehat{D}_{E E^{\prime}}-\frac{1}{2 k} N^{T}\left(\widehat{T}_{E^{\prime} E}-\widehat{T}_{E E^{\prime}}\right) .
$$

\subsubsection{The friction problem}

At each time step, Coulomb's friction conditions can be written as:

- Stick: if $\left\|P \widehat{T}_{E E^{\prime}}\right\|<\mu\left|N^{T} \widehat{T}_{E E^{\prime}}\right|$ then $P \widehat{\dot{D}}_{E E^{\prime}}=0$,

- Slip: if $\left\|P \widehat{T}_{E E^{\prime}}\right\|=\mu\left|N^{T} \widehat{T}_{E E^{\prime}}\right|$ then $\exists \lambda>0$ such that $P \widehat{\dot{D}}_{E E^{\prime}}=-\lambda \widehat{T}_{E E^{\prime}}$.

These conditions can be simply written as:

- Stick: $\left\|G_{t}\right\| \geq \mu\left|N^{T} \widehat{T}_{E E^{\prime}}\right|$,

- Slip: $\left\|G_{t}\right\|<\mu\left|N^{T} \widehat{T}_{E E^{\prime}}\right|$,

where the slip indicator $G_{t}$ is the vector:

$$
G_{t}=\frac{k}{2} P \widehat{\dot{D}}_{E E^{\prime}}-\frac{1}{2} P\left(\widehat{T}_{E^{\prime} E}-\widehat{T}_{E E^{\prime}}\right) .
$$

\subsubsection{Resolution}

The resolution is carried out by projecting the solution of the previous linear stage onto the contact and friction conditions following the search directions:

$$
\begin{aligned}
& \left(\widehat{T}_{E E^{\prime}}-T_{E E^{\prime}}\right)=k\left(\widehat{\dot{W}}_{E E^{\prime}}-\dot{W}_{E E^{\prime}}\right), \\
& \left(\widehat{T}_{E^{\prime} E}-T_{E^{\prime} E}\right)=k\left(\widehat{\dot{W}}_{E^{\prime} E}-\dot{W}_{E^{\prime} E}\right) .
\end{aligned}
$$

The status (Open, Contact, Stick or Slip) of each point of the interface is obtained explicitly since the indicators $c_{n}$ and $G_{t}$ can be derived from the previous solution using the search directions. An implicit time integration scheme is used to calculate the contact indicator $c_{n}$ using an expression in terms of the displacements (see Eq. 15):

$$
\widehat{D}_{E E^{\prime}}^{(t+1)}=\widehat{D}_{E E^{\prime}}^{(t)}+\Delta t \widehat{\dot{D}}_{E E^{\prime}}^{(t+1)} .
$$


The initial condition of the time integration is used to take into account the initial gap. Thus, the contact indicator is written as:

$$
c_{n}=\frac{1}{2 \Delta t} N^{T} \widehat{D}_{E E^{\prime}}-\frac{1}{2 k} N^{T}\left(\widehat{T}_{E^{\prime} E}-\widehat{T}_{E E^{\prime}}\right),
$$

in order to obtain, using the time integration (Eq. 19) and the search directions (Eq. 17 and 18):

$$
\begin{aligned}
c_{n(t+1)} & =\frac{1}{2 \Delta t} N^{T} \widehat{D}_{E E^{\prime}}^{(t+1)}-\frac{1}{2 k} N^{T}\left(\widehat{T}_{E^{\prime} E}^{(t+1)}-\widehat{T}_{E E^{\prime}}^{(t+1)}\right) \\
& =\frac{1}{2} N^{T} \widehat{\dot{D}}_{E E^{\prime}}^{(t+1)}-\frac{1}{2 k} N^{T}\left(\widehat{T}_{E^{\prime} E}^{(t+1)}-\widehat{T}_{E E^{\prime}}^{(t+1)}\right) \\
& +\frac{1}{2 \Delta t} N^{T} \widehat{D}_{E E^{\prime}}^{(t)} \\
& =\frac{1}{2} N^{T} \dot{D}_{E E^{\prime}}^{(t+1)}-\frac{1}{2 k} N^{T}\left(T_{E^{\prime} E}^{(t+1)}-T_{E E^{\prime}}^{(t+1)}\right) \\
& +\frac{1}{2 \Delta t} N^{T} \widehat{D}_{E E^{\prime}}^{(t)} .
\end{aligned}
$$

So

$$
c_{n(t+1)}=\frac{1}{2} N^{T} \dot{D}_{E E^{\prime}}^{(t+1)}-\frac{1}{2 k} N^{T}\left(T_{E^{\prime} E}^{(t+1)}-T_{E E^{\prime}}^{(t+1)}\right)+\frac{1}{2 \Delta t} N^{T} \widehat{D}_{E E^{\prime}}^{(t)} .
$$

Thus, the contact indicator is calculated incrementally from the known solution of the previous global stage. The slip indicator $G_{t}$ (Eq. 16) is calculated explicitly using the search directions (Eq. 17 and 18):

$$
G_{t}=\frac{k}{2} P \dot{D}_{E E^{\prime}}-\frac{1}{2} P\left(T_{E^{\prime} E}-T_{E E^{\prime}}\right) .
$$

\subsection{Properties of the iterative scheme}

As a conclusion, this iterative scheme presents the following properties:

- the strategy gives an approximation of the solution, over the complete time interval, at each iteration,

- the problems that must be solved on each substructure, at each time step and each iteration (12), can have a large size, depending on the way the assembly is decomposed and on the mesh size. However, It is important to notice that the involved operators do not depend on the interface parameters and on the contact statuses. Thus they can be factorized once during the initialization. This reduces the numerical cost of each iteration. 
- when frictional contact is involved, the resolution of the local stage is completely explicit and does not require any iterative process,

- as any Augmented Lagrangian type scheme, the convergence is not rapid but the algorithm is very robust and the iterations are not very costly. The solution that is obtained when convergence is reached is not affected by the parameter $k$, which affects only the rate of convergence

- The quality of the solution is measured using an error indicator that gives an energy norm of the distance between two successive solutions $\widehat{s}$ and $s_{n}$. The expression of this error indicator is given in [12]

- For contact and friction problems where discontinuities can occur, the resolution strategies can be very sensitive to the time and space discretization. The LATIN method acts as a regularization method and present a smooth convergence. This property has already been shown for buckling problems and is presented in [15].

\section{Taking into account structural changes}

\subsection{Principles of multiple resolution}

In order to calculate the influence of the design parameters, an multiple resolution strategy using the LATIN method has been implemented [11]. During the design process, several configurations, corresponding to different values of the sets of design parameters, must be simulated. Thus, some very similar calculations must be carried out in order to get the solutions corresponding to the different sets of design parameters. Multiple resolution means that, the similarity of the problem is used in order to reduce the computational costs. The numerical cost is expected to be less than the cost of one computation multiplied by the number of studied configurations.

Our strategy consists in accelerating the calculation of one solution by using another solution as an initialization. Using the LATIN method, the iterative resolution for one set of parameters is initialized by the solution calculated for 
another set. This is done very simply by changing the parameters between two successive iterations. The strategy is described in figure 4. As each iteration of the LATIN method generates a solution on the whole time interval, the initialization overacts on the whole time interval. Due to the strong mechanical content of this initialization, the number of iterations needed to reach convergence for this new calculation is reduced. Indeed, if the two solutions are close, the number of iterations can be very small.

So far, the parameters whose influence have been taken into account are associated to the connection behavior: gaps, pre-strains, friction coefficient. For such design parameters, this multiple resolution strategy leads to a great reduction of computational costs as shown in [12]. In the example presented in this last paper, the initialization solution was the one computed just before. An optimization of the choice of the initialization is presented in [35].

In the present work, the same multiple resolution strategy is used to study cases where the design parameters are the presence of the different fasteners (bolts, screws, ... ). In order to study the influence of missing fasteners on the behavior, a first iterative calculation, including all the fasteners, is performed until convergence is reached. Then, the substructures that correspond to missing fasteners (bodies, heads, nuts, ... ) are de-activated. De-activation means that the substructures stay in the model but do not interact with the neighboring interfaces. Then, the iterative process is continued until convergence to the solution of this new configuration. As the iterative process is continued, the calculation of this last solution is then initialized by the solution of the previous situation where the fastener is present.

De-activating a substructure is a very simple action. The linear stage of the LATIN method is not performed on such structures. It only returns a null force field

$$
F_{E \Gamma}=0,
$$

and the velocity field associated by the search direction:

$$
\dot{U}_{E \Gamma}=\widehat{\dot{U}}_{E \Gamma}-\frac{1}{k}\left(F_{E \Gamma}-\widehat{F}_{E \Gamma}\right)=\widehat{\dot{U}}_{E \Gamma}+\frac{1}{k} \widehat{F}_{E \Gamma} .
$$


The next iterations, until convergence, calculate the new solution for the assembly without the missing fasteners, as if the interfaces between the missing substructures and the rest of the assembly were prescribed null force interfaces.

At the end of this second calculation, the concerned substructures are reactivated and some others are de-activated in order to study the influence of another missing fastener in a third calculation.

\subsection{Improvements}

As presented in the examples at the end of this paper, it is often observed that the solution for one missing fastener is closer to the solution with no missing fasteners than the solution for any other missing fastener. In our activation deactivation process during the iterations, the calculation for one missing fastener is naturally initialized by the solution of the previous calculated solution that often corresponds to another missing fastener.

In order to initialize each new calculation with the solution obtained for the assembly featuring all its fasteners, this last solution is computed first and then saved. A solution consists only in the velocity and force vectors on each

interface $s=\bigcup_{\Gamma_{E E^{\prime}}}\left(\dot{U}_{E E^{\prime}}, F_{E E^{\prime}}, \dot{U}_{E^{\prime} E}, F_{E^{\prime} E}\right)$, at each time step. Indeed, as the behavior of the substructures is linear, the internal solutions can easily be reconstructed from the boundary fields. Such a solution corresponds to a small amount of stored data. Each time a new calculation is started, i.e. each time fastener substructures are deactivated or reactivated, this solution is restored: forces and velocity vectors of the linear stage are set back to the stored values before continuing the iterations. Thus, the new calculation is initialized by the stored solution. The efficiency of this improvement based on the selection of the initial solution is shown on the next example.

\section{Example}

In the following examples, only linear material behavior is considered. This means that the only non-linearities are unilateral contact and friction. One only 
seeks for the effects of missing connecting elements on the distribution of forces and the loading conditions on the most loaded element. These effects are studied under the assumption of small deformations and displacements. From these results a local re-analysis can be conducted in order to simulate the behavior of the most loaded connector. This last simulation can include non-linear behavior such as plasticity, damage and failure. Such simulations are not presented here.

As announced in the introduction, the proposed examples present conformal meshes on the contact zones. The meshes are obtained from CAD models using an automatic procedure. The CAD model does not include the connecting elements (bolts or screws) but only their localization and their type. A geometrical patch is automatically introduced where an element is located and conformal meshes are automatically built on this patch.

\subsection{First example : bolted connection}

This example deals with the assembly of three aluminium plates fastened by two steel bolts (figure 5). The assembly is submitted to traction. One seeks for the solution for the case where the two bolts are present in the assembly and the solutions in which each one of the bolts is successively missing. For symmetry reasons, only one fourth of the assembly is modeled (figure 6). A coarse mesh (7,819 dof) and a fine one (169,380 dof) are used for his example. Figure 7 presents the fine mesh and the distribution of von Mises equivalent stress for the three studied configurations. Configuration \#1 includes both the bolts. Left bolt is deactivated in configuration \#2 and right bolt is deactivated in configuration \#3.

Figure 8 shows the evolution of the LATIN method convergence indicator along iterations. One can easily notice the strong discontinuity of the evolution whenever substructures are activated or dis-activated. The number of iterations needed to reach convergence for the second calculation is only $40 \%$ of the number needed for the first calculation. The strong effect of the initialization is clearly shown here.

On the contrary, the number of iterations of the third calculation is the same 
as for the first one, when no solution selection technique is used. On such a two bolt structure, it is obvious that the solution of the second configuration can not be a useful initialization for the third one. When this last calculation is initialized with the solution of the first calculation (see figure 9), acceleration is observed. The efficiency of the acceleration is the same as the one observed for the second calculation.

Table 1 presents the number of iterations for each calculated configuration and for three different used strategies:

- without multi-resolution acceleration: one different calculation for each configuration

- multi-resolution without initial solution selection: each calculation is initialized by the calculation of the previous one,

- multi-resolution with initial solution selection: calculations \#2 and \#3 are initialized by the solution of calculation \#1 (two bolts present).

\section{Second example : wheel-shaft assembly}

This example deals with the assembly of a gear wheel on a hollow shaft using eight pre-strained screws (figure 10). The hollow shaft is cantilevered on one end and the wheel is submitted to a force that corresponds to the force applied on the tooth of an helical gear.

Figure 11 presents the distribution of the von Mises equivalent stress for each of the nine calculated configurations. Configuration \#1 is the assembly with its eight screws. For each configurations \#2-9, one of the screws is missing.

Figure 8 shows the evolution of the LATIN method convergence indicator along iterations. One can easily notice the strong discontinuity of the evolution whenever substructures corresponding to screws are activated or deactivated. The number of iterations needed to reach convergence for configuration \#1 is nearly the same as the total number of iterations needed to get the solutions for configurations \#2-9 (see figure 12). 


\section{Conclusions}

An accelerated strategy for the quick calculation of the influence of missing fasteners on the behavior of connections was presented. It is based on the use of a decomposition of the assembly into substructures and interfaces and on an iterative resolution scheme based on the LATIN Method. The reusing of stored solutions for initialization allows a strong reduction of the number of calculated iterations and so of the computational costs. The model that is stored in the computer stays the same during the whole process. Structures corresponding to missing fasteners are simply deactivated. This limits the data exchanges. Moreover, the main operators remain constants during the process and are factorized

only once at the beginning. The proposed examples of assemblies showed the efficiency of the approach and some possible improvements.

\section{References}

[1] W. Barrois, Stresses and displacements due to load transfer by fasteners in structural assemblies, Eng. Frac. Mech. 10 (1) (1978) 115-176.

[2] S. Yen, Multirow joint fastener load investigation, Comput. Struct. 9 (5) (1978) 483-488.

[3] F. Wei-Xun, Load distribution of multi-fastener laminated composite joints, Int. J. Sol. Struct. 30 (21) (1993) 3013-3023.

[4] J. Ekh, J. Schon, Finite element modeling and optimization of load transfer in multi-fastener joints using structural elements, Compos. Struct. 82 (2) (2008) 245-256.

[5] L. Gaul, R. Nitsche, The Role of Friction in Mechanical Joints, App. Mech. Rev. 54 (2) (2001) 93-106.

[6] N. Pai, Three-dimensional finite element analysis of threaded fastener loosening due to dynamic shear load, Eng. Fail. Anal. 9 (4) (2002) 383-402. 
[7] J. Yang, F.-K. Chang, Detection of bolt loosening in CC composite thermal protection panels: I. Diagnostic principle, Smart Mater. Struct. 15 (2) (2006) 581-590.

[8] T. Jaglinski, A. Nimityongskul, R. Schmitz, R. S. Lakes, Study of Bolt Load Loss in Bolted Aluminum Joints, J. Eng. Mater. T. 129 (1) (2007) $48-54$.

[9] M. Zhang, Y. Jiang, C.-H. Lee, Finite Element Modeling of Self-Loosening of Bolted Joints, J. Mech. Design 129 (2) (2007) 218-226.

[10] W. F. Stanley, M. a. McCarthy, V. P. Lawlor, Measurement of load distribution in multibolt composite joints in presence of varying clearance, Plast. Rubber Compos. 31 (9) (2002) 412-418.

[11] P. Boucard, L. Champaney, A suitable computational strategy for the parametric study of problems with multiple contact, Int. J. for Numer. Meth. Engrg. 57 (9) (2003) 1259-1281.

[12] L. Champaney, P. Boucard, S. Guinard, Adaptive multi-analysis strategy for contact problems with friction. Application to aerospace bolted joints, Comput. Mech. 2 (42) (2008) 305-316.

[13] L. Champaney, Computational strategy for taking geometrical uncertainties into account in structural assemblies, in: Proc. WCCM8 - 8th World Congress on Computational Mechanics, Venice, Italy, June, 2008.

[14] A. Shimada, K. Urabe, K. Yoshihiro, J. Takahashi, K. Kageyama, Detection of missing fastener based on vibration mode analysis using fiber Bragg Grating (FBG) sensors, in: Amr M. Baz (Ed.), Smart Structures and Materials 2003: Smart Structures and Integrated Systems Proceedings of SPIE, Vol. 5056, 2003, pp. 312-318.

[15] P. Ladevèze, Nonlinear Computational Structural Mechanics - New Approaches and Non-Incremental Methods of Calculation, Springer Verlag, 1999. 
[16] L. Champaney, J. Cognard, P. Ladevèze, Modular analysis of assemblages of three-dimensional structures with unilateral contact conditions, Comput. Struct. 73 (1-5) (1999) 249-266.

[17] D. Odièvre, P.-A. Boucard, F. Gatuingt, A parallel, multiscale domain decomposition method for the transient dynamic analysis of assemblies with friction, Comput. Methods Appl. Mech. Engrg. 199 (21-22) (2010) 12971306.

[18] Z. Zhong, J. Mackerle, Static contact problems. A review, Engrg. Comput. 9 (1) (1992) 3-37.

[19] F. Lebon, Contact problems with friction: models and simulations, Simul. Model. Pract. Theory 11 (5-6) (2003) 449-463.

[20] P. Wriggers, Computational contact mechanics, Springer, 2006.

[21] N. Kikuchi, Penalty/finite element approximations of a class of unilateral contact problems, in: Penalty-finite element methods in mechanics: presented at the Winter Annual Meeting of the American Society of Mechanical Engineers, Phoenix, Arizona, November 14-19, 1982, ASME, 1982.

[22] F. Armero, Formulation and analysis of conserving algorithms for frictionless dynamic contact/impact problems, Comput. Methods Appl. Mech. Engrg. 158 (3-4) (1998) 269-300.

[23] P. Alart, A. Curnier, A mixed formulation for frictional contact problems prone to Newton like solution methods, Comput. Methods Appl. Mech. Engrg. 92 (3) (1991) 353-375.

[24] J. S. Arora, A. I. Chahande, J. K. Paeng, Multiplier methods for engineering optimization, Int. J. for Numer. Meth. Engrg. 32 (7) (1991) 1485-1525.

[25] A. Klarbring, Mathematical programming and augmented Lagrangian methods for frictional contact problems, in: C. A. (Ed.), Proceedings Contact Mechanics International Symposium, October, Presses Polytechniques et Universitaires Romandes, 1992, p. 79. 
[26] B. Radi, Treatment of the frictional contact via a Lagrangian formulation, Mathematical and Computer Modelling 28 (4-8) (1998) 407-412.

[27] P. Chabrand, Various numerical methods for solving unilateral contact problems with friction, Math. Comput. Model. 28 (4-8) (1998) 97-108.

[28] J. Koko, Uzawa block relaxation domain decomposition method for the two-body contact problem with Tresca friction, Comput. Methods Appl. Mech. Engrg. 198 (3-4) (2008) 420-431.

[29] K. Arrow, L. Hurwicz, H. Uzawa, Studies in linear and non-linear programming, Stanford University Press, Stanford, California, 1958.

[30] J. Simo, T. Laursen, An augmented lagrangian treatment of contact problems involving friction, Comput. Struct. 42 (1) (1992) 97-116.

[31] P. Avery, C. Farhat, The FETI family of domain decomposition methods for inequality-constrained quadratic programming: Application to contact problems with conforming and nonconforming interfaces, Comput. Methods Appl. Mech. Engrg. 198 (21-26) (2009) 1673-1683.

[32] J. Danek, I. Hlavcek, J. Nedoma, Domain decomposition for generalized unilateral semi-coercive contact problem with given friction in elasticity, Math. and Comput. Simulat. 68 (3) (2005) $271-300$.

[33] Z. Dostal, D. Horak, R. Kucera, J. Haslinger, J. Dobias, S. Ptak, Feti based algorithms for contact problems: scalability, large displacements and 3d coulomb friction, Comput. Methods Appl. Mech. Engrg. 194 (2005) 295-409.

[34] R. Krause, A non-smooth multiscale method for solving frictional twobody contact problems in $2 \mathrm{~d}$ and $3 \mathrm{~d}$ with multigrid efficiency, SIAM J. Sci. Comput. 31 (2) (2009) 1399-1423.

[35] P. Boucard, S. Buytet, P. Guidault, A multiscale strategy for structural optimization, Int. J. for Numer. Meth. Engrg. 78 (1) (2009) 101-126. 


\section{List of Figures}

1 Decomposition of the structure . . . . . . . . . . 23

2 Compatible meshes on the interfaces . . . . . . . . . . . 23

3 LATIN scheme . . . . . . . . . . . . . . . . . . 24

4 Multiple resolution algorithm . . . . . . . . . . . . . 24

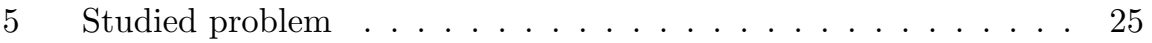

6 Studied model, dimensions in millimeters _ . . . . . . . . 25

$7 \quad$ Fine Mesh and Von Mises equivalent stress for the three studied configurations $(\mathrm{MPa}) \ldots \ldots \ldots \ldots \ldots \ldots \ldots$

8 Error Indicator along the iterations when each calculation is initialized by the solution of the previous one (coarse mesh). . . . . 26

9 Error Indicator along the iterations when the second and third calculations are initialized by the solution of the first one (coarse

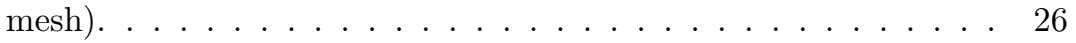

10 Studied problem: eight bolt connection between a wheel and a

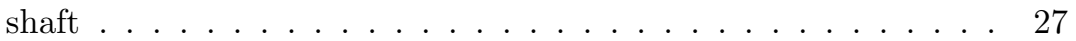

11 Von Mises equivalent stress on each one of the nine calculated configuration $(\mathrm{MPa}) \ldots \ldots \ldots \ldots \ldots$

12 Error indicator for the complete simulation (nine calculations). . 29

\section{List of Tables}

1 Computational costs (numbers of iterations) with or without improvement based on the selection of the initial solution . . . . . 27 


\section{Figures}

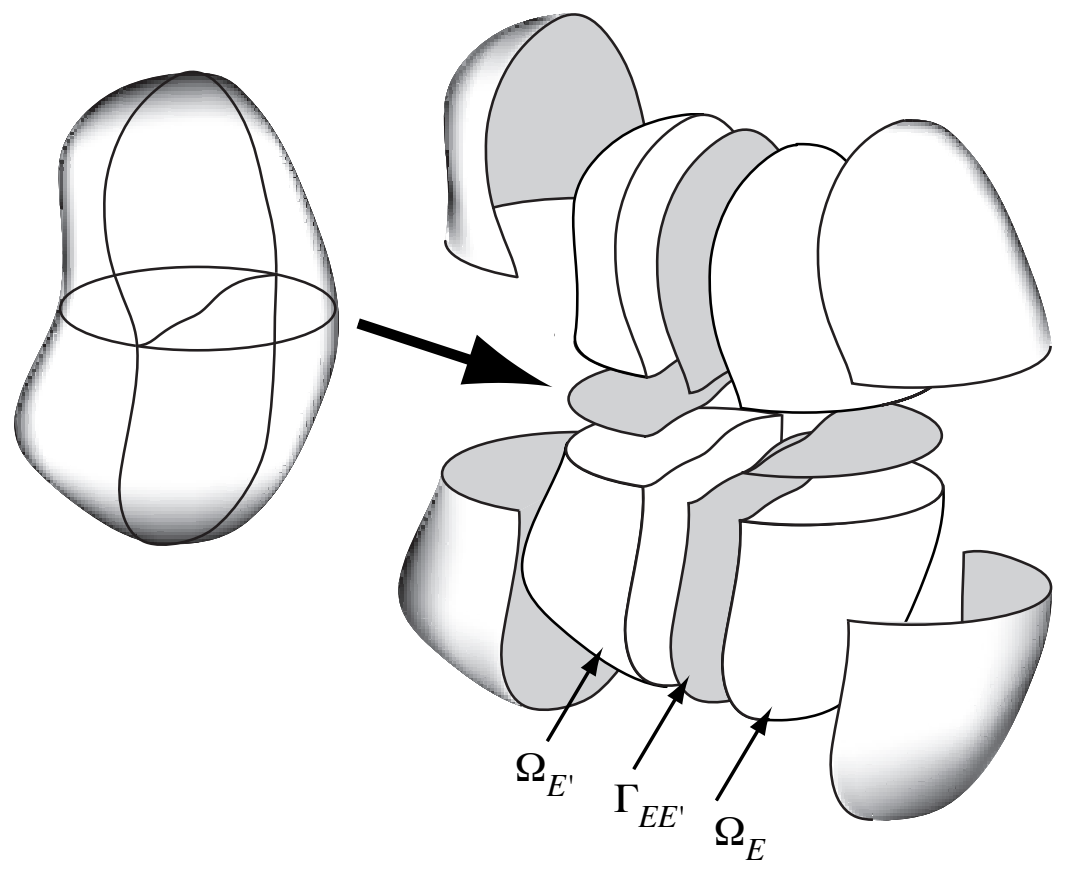

Figure 1: Decomposition of the structure

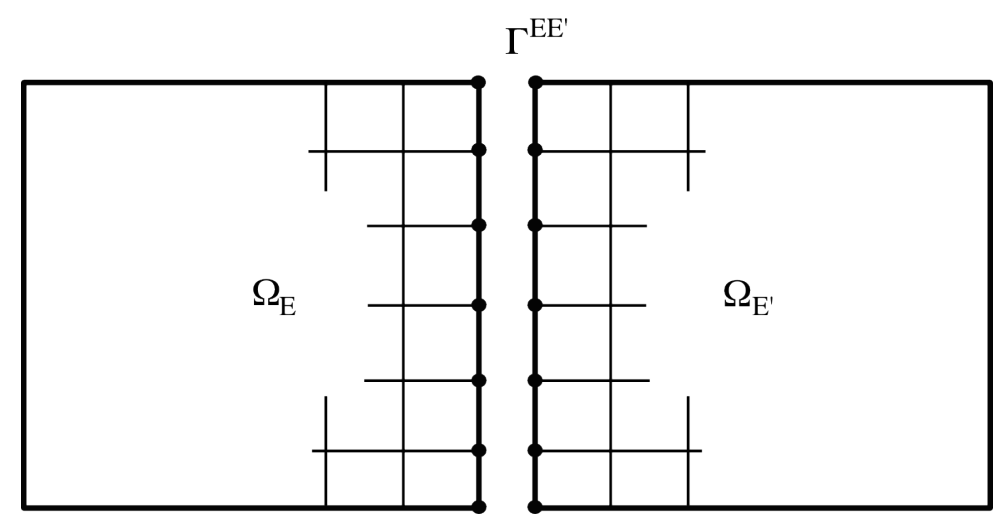

Figure 2: Compatible meshes on the interfaces 


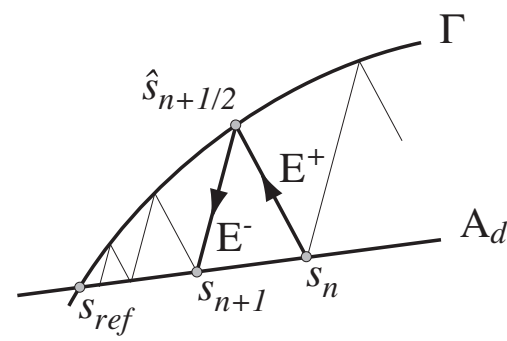

Figure 3: LATIN scheme

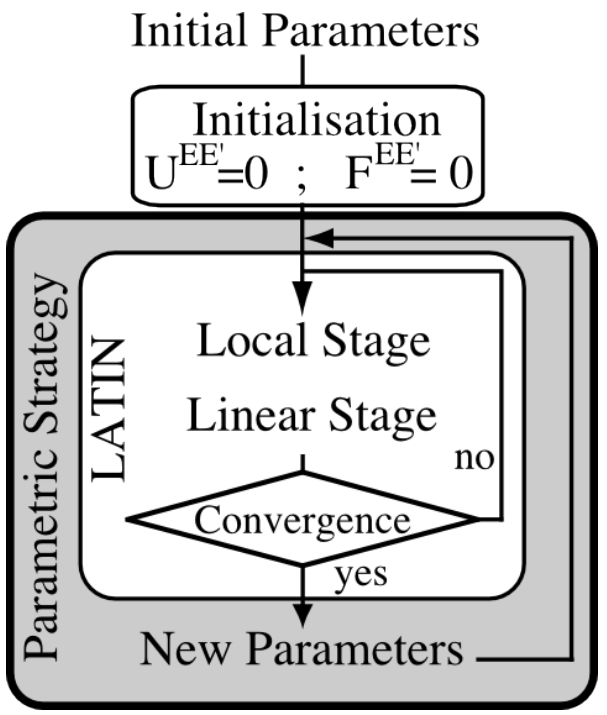

Figure 4: Multiple resolution algorithm 


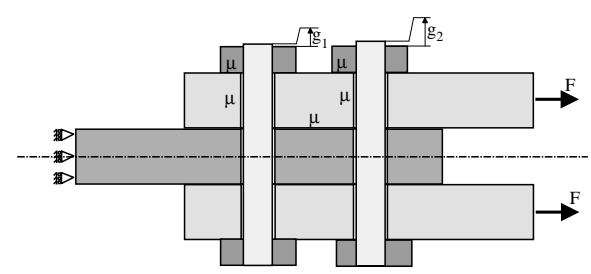

Figure 5: Studied problem

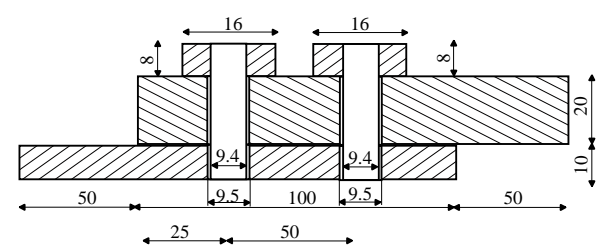

Figure 6: Studied model, dimensions in millimeters

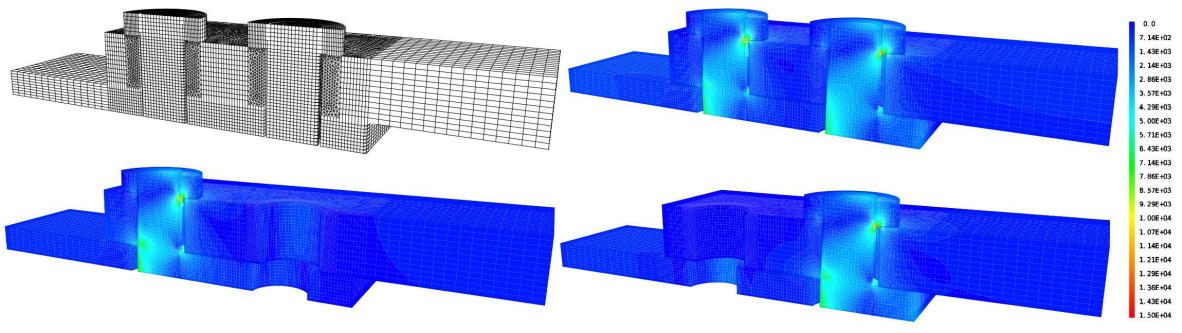

Figure 7: Fine Mesh and Von Mises equivalent stress for the three studied configurations $(\mathrm{MPa})$ 


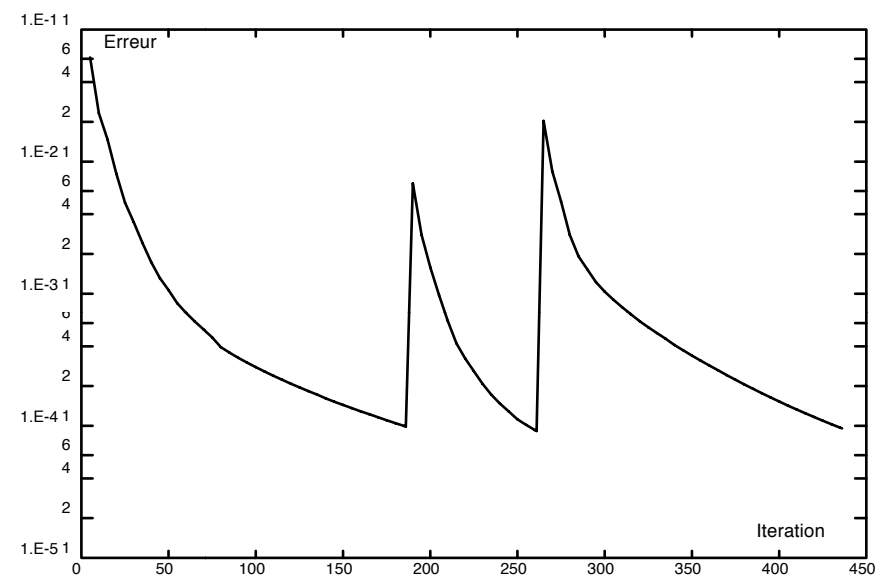

Figure 8: Error Indicator along the iterations when each calculation is initialized by the solution of the previous one (coarse mesh).

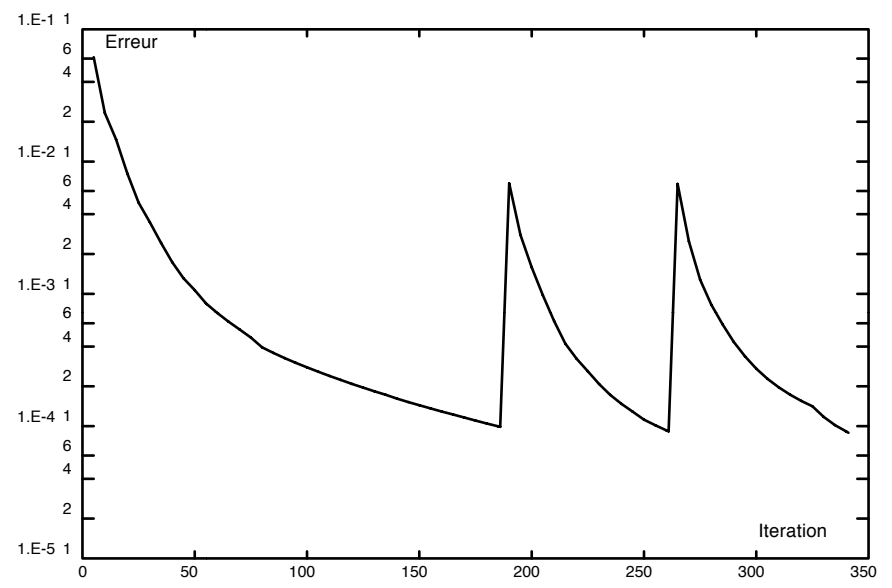

Figure 9: Error Indicator along the iterations when the second and third calculations are initialized by the solution of the first one (coarse mesh).

tables 


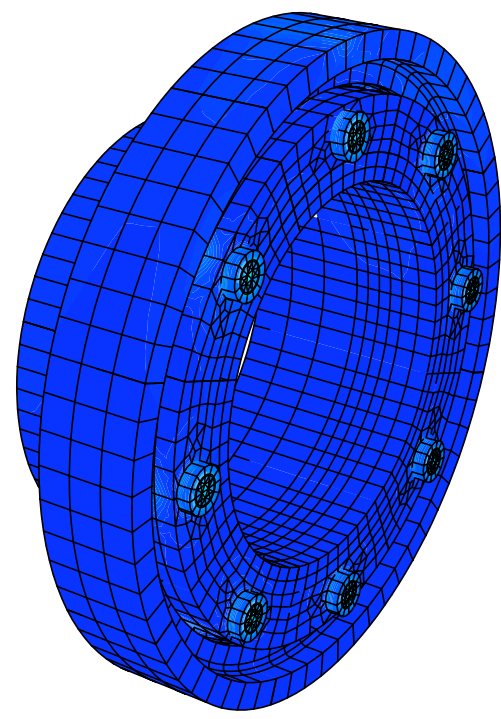

Figure 10: Studied problem: eight bolt connection between a wheel and a shaft

\begin{tabular}{|l|c|c|c|c|c|c|}
\hline Mesh & \multicolumn{3}{|c|}{ Coarse (7,812 dof) } & \multicolumn{3}{c|}{ Fine (169,380 dof) } \\
\hline Type & direct & w/o imp. & w imp. & direct & w/o imp. & w imp. \\
\hline Calculation \#1 & 186 & 186 & 186 & 255 & 255 & 255 \\
Calculation \#2 & 190 & 75 & 75 & 262 & 55 & 55 \\
Calculation \#3 & 192 & 175 & 80 & 268 & 85 & 55 \\
\hline Total & 568 & 436 & 339 & 785 & 395 & 365 \\
\hline
\end{tabular}

Table 1: Computational costs (numbers of iterations) with or without improvement based on the selection of the initial solution 

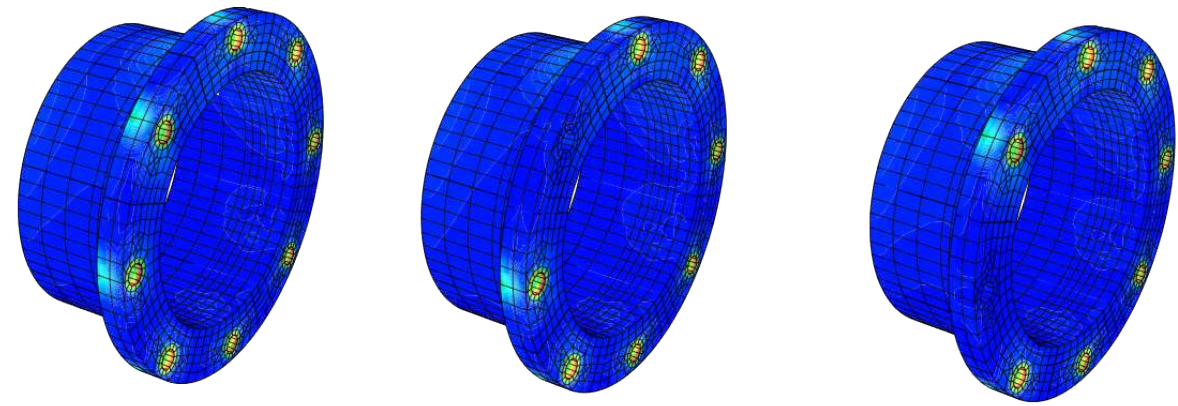

0. 71

5. 4

10.

15.

19.

24.
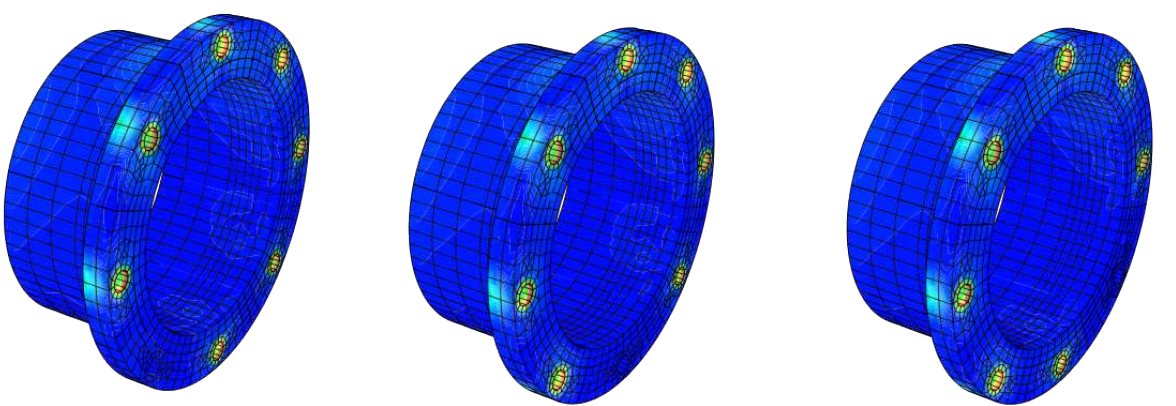

29.

33.

38.

43.

48.

52.

57.

62.
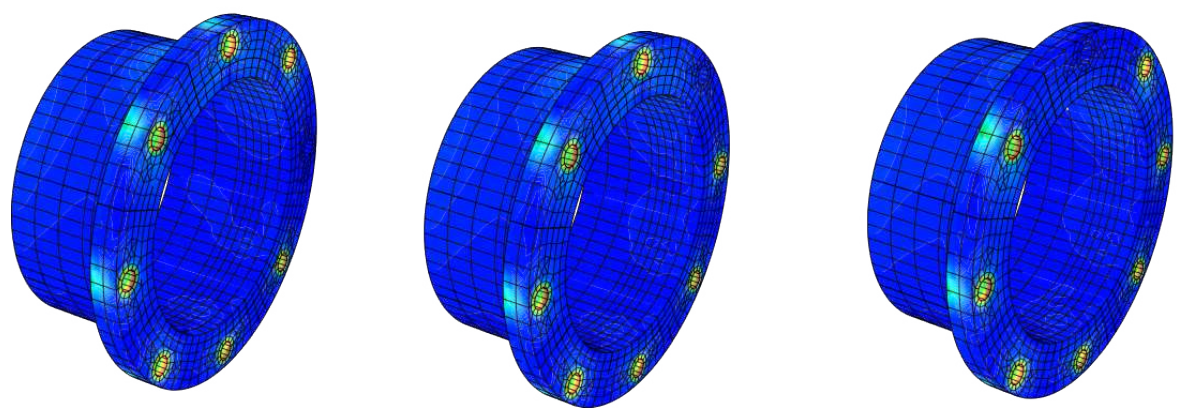

66.

71.

76.

80.

85.

90.

94.

99.

Figure 11: Von Mises equivalent stress on each one of the nine calculated configuration (MPa) 


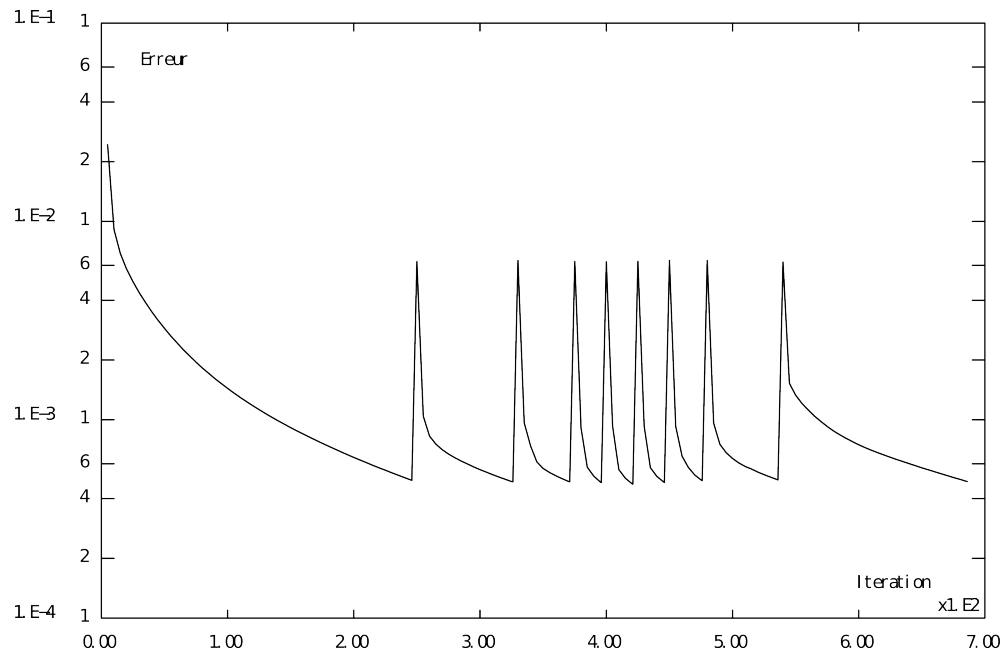

Figure 12: Error indicator for the complete simulation (nine calculations). 